\title{
Integrating Sphere Microscopy for Direct Absorption Measurements of Single Nanostructures
}

Sander A. Mann, ${ }^{\dagger}$ Beniamino Sciacca, ${ }^{\dagger}$ Yunyan Zhang, ${ }^{\ddagger}$ Jia Wang, ${ }^{\dagger}$ Evgenia Kontoleta, ${ }^{\dagger}$ Huiyun Liu, ${ }^{\dagger}$ and Erik C. Garnett* ${ }^{\dagger}$

${ }^{\dagger}$ Center for Nanophotonics, AMOLF, Science Park 104, 1098 XG Amsterdam, The Netherlands

${ }^{*}$ Department of Electronic and Electrical Engineering, University College London, London WC1E 7JE, United Kingdom

\section{Supporting Information}

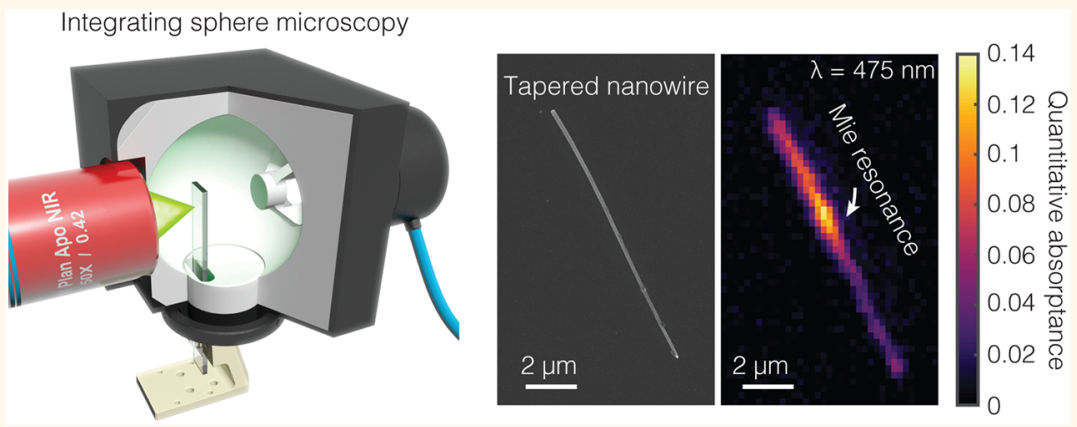

ABSTRACT: Nanoscale materials are promising for optoelectronic devices because their physical dimensions are on the order of the wavelength of light. This leads to a variety of complex optical phenomena that, for instance, enhance absorption and emission. However, quantifying the performance of these nanoscale devices frequently requires measuring absolute absorption at the nanoscale, and remarkably, there is no general method capable of doing so directly. Here, we present such a method based on an integrating sphere but modified to achieve submicron spatial resolution. We explore the limits of this technique by using it to measure spatial and spectral absorptance profiles on a wide variety of nanoscale systems, including different combinations of weakly and strongly absorbing and scattering nanomaterials ( $\mathrm{Si}$ and GaAs nanowires, Au nanoparticles). This measurement technique provides quantitative information about local optical properties that are crucial for improving any optoelectronic device with nanoscale dimensions or nanoscale surface texturing.

KEYWORDS: absorption spectroscopy, nanowires, quantitative spectroscopy, Mie resonances, nanoparticles, plasmon resonance

A

number of techniques to measure quantitative absorptance on single nanostructures exist, ${ }^{1-3}$ such as photothermal spectroscopy, ${ }^{4-7}$ extinction (modulation) spectroscopy, ${ }^{8-10}$ and scattered field interferometry. ${ }^{11}$ Although some of these techniques are extremely sensitive and can even measure single molecules, ${ }^{5,12}$ they also rely on at least one of the following assumptions: (i) all absorbed power turns into heat; (ii) the nanoparticle does not scatter light; or (iii) it interacts with light as a dipole. Hence, to date, there is no generally applicable method available for quantitative absorption measurements of nanostructures, even though, for instance, nanostructured semiconductor and plasmonic hotelectron optoelectronic devices ${ }^{13,14}$ require such a technique to quantify their performance.

Here, we show that by combining an integrating sphere with a microscope objective with an ultralong working distance, we can achieve submicron spatial resolution inside the integrating sphere. As a result, quantitative local absorptance measurements can be obtained, even on single nanostructures. We have recently used this method to measure strongly absorbing nanowires either to verify core-shell nanowire antenna properties or for internal quantum efficiency measurements. ${ }^{15,16}$ Now, we detail the working mechanism and explore the limits of the technique using weakly absorbing but strongly scattering structures (silicon nanowire and a large gold nanoparticle), a weakly absorbing and weakly scattering structure (small gold nanoparticle), and a strongly absorbing and strongly scattering structure (GaAs nanowire). Additionally, we demonstrate the spatial resolution with absorption

Received: September 28, 2016

Accepted: January 5, 2017

Published: January 5, 2017 

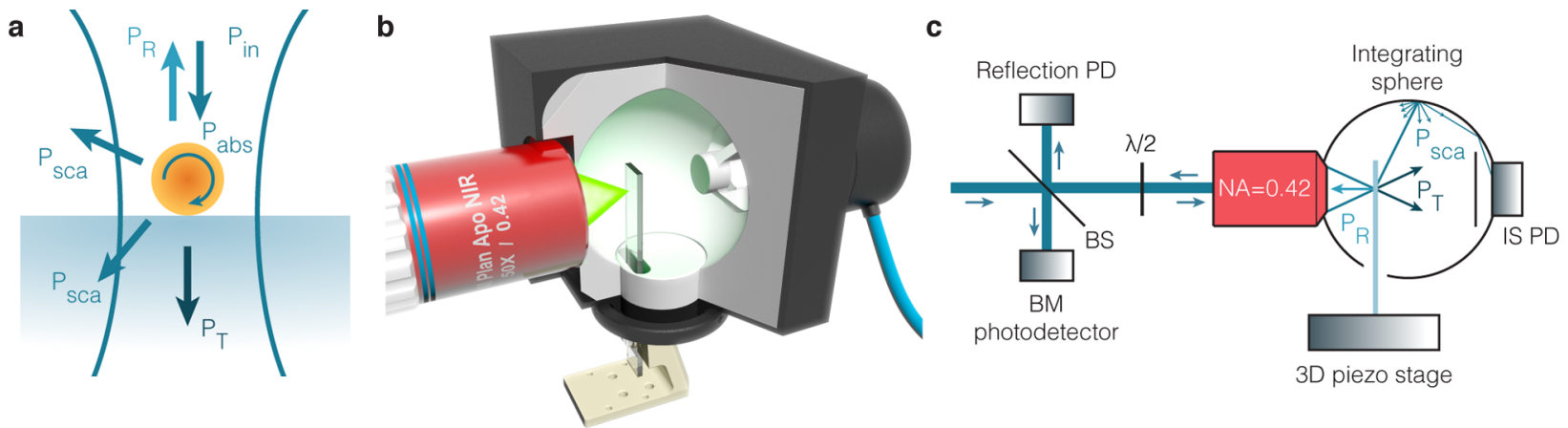

Figure 1. Schematic of the integrating sphere setup. (a) When illuminating a nanoparticle with a focused beam, the nanoparticle takes power from the incident beam and absorbs $\left(P_{\text {abs }}\right)$ or scatters $\left(P_{\text {sca }}\right)$ it. Most of the power is just reflected $\left(P_{\mathrm{R}}\right)$ or transmitted $\left(P_{\mathrm{T}}\right)$. In an integrating sphere, the scattered $\left(P_{\text {sca }}\right)$ and transmitted light $\left(P_{\mathrm{T}}\right)$ are both detected, so that by combining that signal with the reflected signal $\left(P_{\mathrm{R}}\right)$ the absorptance can be determined. (b) Schematic depiction of the combination of microscope objective and integrating sphere. The objective focuses light on the sample holder inside the integrating sphere, and the photodetector is behind a baffle on the backside of the integrating sphere. The sample extends outside of the integrating sphere through a small port, where the sample is mounted on a piezoelectric stage for sample movement. (c) Schematic diagram for the experimental setup, including reflection photodetector (PD) and half-wave plate $(\lambda / 2)$ to control the polarization. BS stands for beam splitter and BM for beam monitor, which we use to account for fluctuations in laser power. NA stands for the numerical aperture of the objective.

maps on a highly tapered nanowire, where resonances can be seen moving along the length as a function of wavelength.

Single nanoparticle spectroscopy relies on illumination with a focused beam to enhance the interaction with the nanoparticle. When illuminating a nanoparticle, part of the power will be absorbed or scattered by the nanoparticle $\left(P_{\mathrm{abs}}\right.$ and $P_{\text {sca }}$; see Figure 1a). Additionally, even for a focused beam, the majority of the incident power will typically not interact with the nanoparticle and be either transmitted or reflected $\left(P_{\mathrm{T}}\right.$ and $\left.P_{\mathrm{R}}\right)$. Just as with the standard integrating sphere method, ${ }^{17,18}$ integrating sphere microscopy relies on determining the scattered and transmitted light. Due to the diffuse and highly reflective surface of an integrating sphere, this transmitted and scattered light quickly randomizes and covers the inside of the integrating sphere with equal intensity. This homogenized intensity also reaches a photodetector attached to the integrating sphere, which thus detects a power proportional to the total transmitted and scattered power that is independent of the exact scattering pattern. Through a reference measurement (which has no absorbing structure), the absolute transmitted and scattered power can then be determined (see Supporting Information for more details on the setup). Note that no distinction can be made between transmitted and scattered power inside the integrating sphere, and it is therefore not possible to determine the extinction cross section simultaneously.

To combine integrating sphere measurements with submicron spatial resolution, we combined an integrating sphere with a microscope objective. ${ }^{15,16,19}$ This microscope objective focuses the light on the sample inside the integrating sphere (see Figure $1 \mathrm{~b}$ ). The sample is mounted on a 3D piezoelectric stage, which allows scanning of the sample to obtain quantitative local information on the absorptance. The ability to scan the sample or focused spot position is crucial: without scanning, integrating sphere microscopy can only be used in a wide field configuration, which does not allow for quantitative spatial information. ${ }^{19}$

A detector behind a baffle on the backside of the integrating sphere measures most of the transmitted and scattered power; some light will be reflected or backscattered into the microscope objective. This fraction is imaged on a second photodetector by the microscope objective and is therefore also collected (see Figure 1c). Hence, by measuring the scattered, reflected, and transmitted light, the absorbed light can be inferred: $P_{\mathrm{abs}}=1-P_{\mathrm{R}}-P_{\mathrm{T}}-P_{\mathrm{sca}}$. More specifically, for the absorptance, we then obtain

$$
A=1-\frac{R}{R_{\mathrm{ref}}}-\frac{\mathrm{IS}}{\mathrm{IS} \mathrm{ref}_{\mathrm{ref}}}-C
$$

where $R$ and $R_{\text {ref }}$ are the reflection signal and reference measurement and IS and IS ref are the integrating sphere signal and reference measurement. Calibration of the technique is thus extremely simple: it only requires two reference measurements, usually a mirror for $R_{\text {ref }}$ and a small hole in the substrate for $\mathrm{IS}_{\text {ref }}$ (meaning that the beam misses the sample, but the sample is still in the integrating sphere). The experimental setup is discussed in more detail in the Supporting Information. Note that some samples can be strongly backscattering, and it is therefore important to record the reflected power (see Supporting Information for examples).

In eq $1, C$ is an offset that arises from the reflection of the substrate-air interface on the back of the substrate, which the objective collects, but is so far beyond the focal plane that it is not imaged on the photodetector. For glass substrates, it is $\sim 0.03$ (see Supporting Information), but it depends slightly on how much power is transmitted and scattered forward, and a small error is therefore introduced by assuming that it is constant. The accuracy of the technique additionally depends on the detection efficiency of all nonabsorbed light; for example, if some light is scattered but not detected by either photodetector, it will appear as absorption. Based on measurements on nonabsorbing but strongly scattering silica beads, we estimate that $95 \pm 1.2 \%$ of the total scattered power is collected. ${ }^{16}$ Finally, broadband characterization is possible since we use a supercontinuum laser as a light source (400$2000 \mathrm{~nm}$ ), and the integrating sphere inner surface remains Lambertian from 250 to $2500 \mathrm{~nm}$.

\section{RESULTS AND DISCUSSION}

We will now demonstrate this technique by showing local variation in the absorptance of a tapered silicon nanowire. Semiconductor nanowires are important building blocks for 

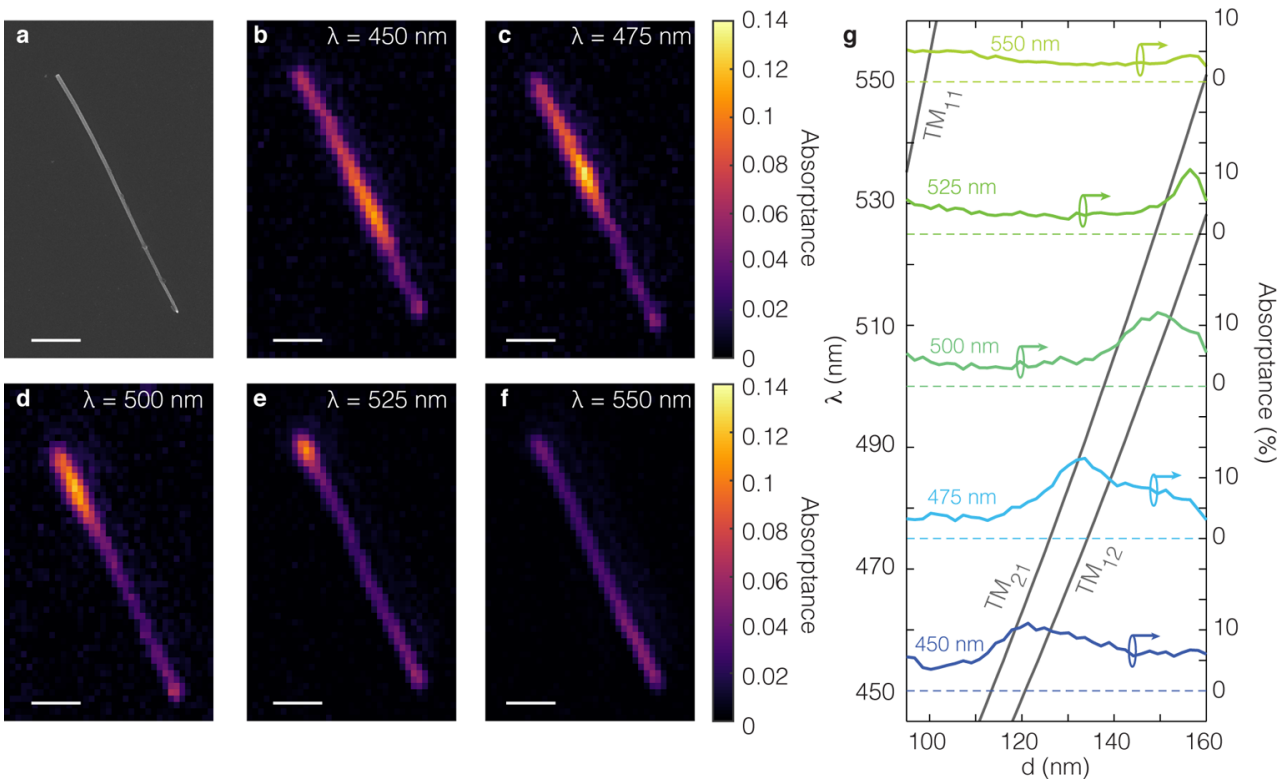

Figure 2. Absorptance maps of a silicon nanowire. (a) SEM image of the silicon nanowire under consideration. Its diameter ranges from 95 (bottom) to $160 \mathrm{~nm}$ (top). The scale bar is $2 \mu \mathrm{m}$. (b-f) Absorptance maps over the same spatial range for different wavelengths. For all maps, the laser polarization is aligned with the long axis of the nanowire. The absorption peak shows how the $\mathbf{T M}_{12}$ and $\mathrm{TM}_{21}$ resonances shift position along the wire as the wavelength increases, and the $T M_{11}$ is seen to appear on the bottom side of the wire. (g) Wavelength dispersion of the resonances of a circular silicon nanowire in vacuum as a function of diameter, with the absorptance spectra at different wavelengths superimposed. The peaks in absorption along the length of the wire clearly correspond to the presence of resonances.
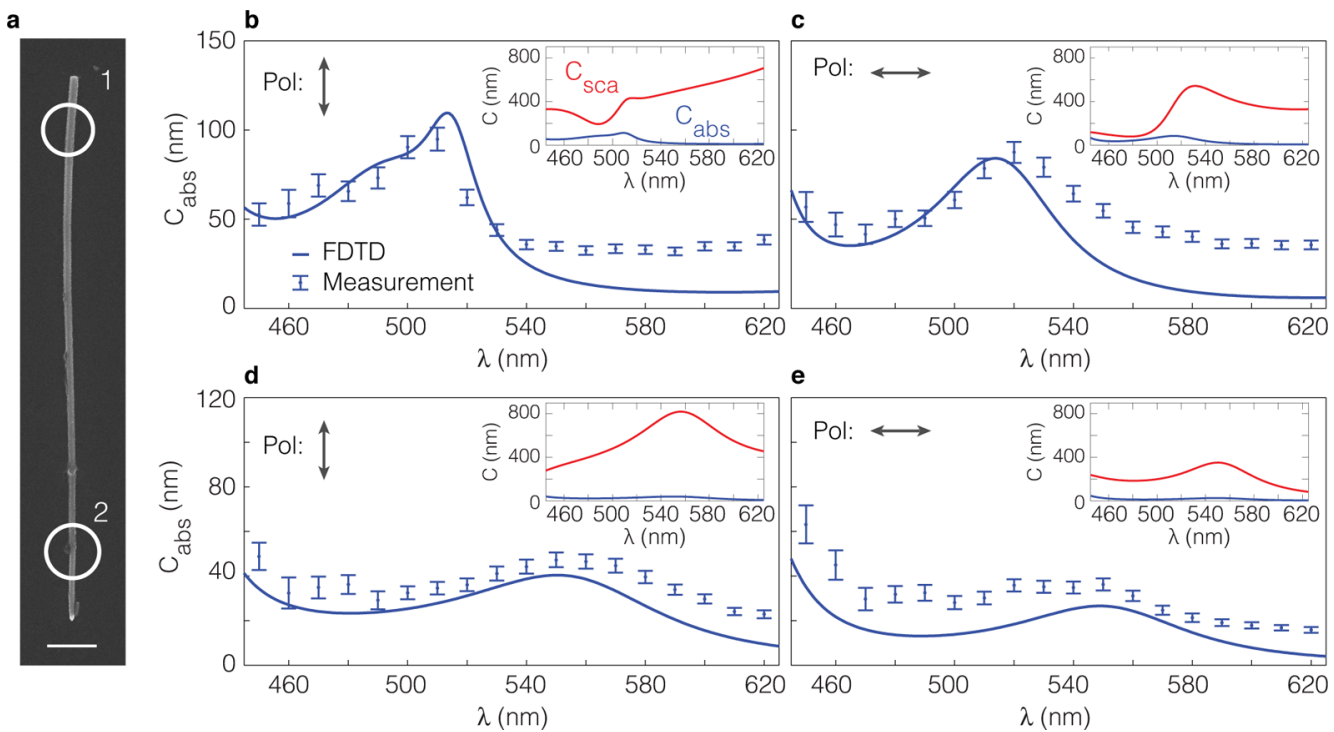

Figure 3. Absorption spectra of a silicon nanowire. (a) SEM image of the silicon nanowire indicating the positions at which spectra were measured. The scale bar is $1 \mu \mathrm{m}$. (b) Measured absorption cross section of the silicon nanowire at position 1 with the electric field polarized along the nanowire axis, compared to simulations of a nanowire on glass with a diameter of $156 \mathrm{~nm}$ (blue line; see Methods). The inset shows the simulated absorption cross section (blue) compared to the scattering cross section (red), indicating that the wire scatters much more strongly than it absorbs. (c) Same as in panel (b) but with the electric field polarized perpendicular to the axis. (d,e) Same as panels (b) and (c) but measured at position 2 and with $112 \mathrm{~nm}$ diameter nanowire simulation. In $(\mathrm{b}-\mathrm{e})$, the error bars are due to variance in the absorptance and uncertainty in the conversion to cross section.

optoelectronics applications, ${ }^{20}$ including nanowire solar cells, ${ }^{21,22}$ light-emitting diodes, ${ }^{23-25}$ lasers, $^{26}$ and quantum information technology. ${ }^{27}$ Silicon nanowires, in particular, provide a challenging test case for nanoscale absorption measurements because the indirect band gap leads to scattering that can be 2 orders of magnitude stronger than the absorption. Figure 2a shows an scanning electron microscopy (SEM) image of a $10 \mu \mathrm{m}$ long silicon nanowire on a glass substrate. The nanowire is slightly tapered, with a diameter that varies from $160 \mathrm{~nm}$ at the top of the image to $95 \mathrm{~nm}$ at the bottom. Figure $2 \mathrm{~b}$ displays the absorptance $A$ (absorbed fraction of the incident power, $\left.P_{\mathrm{abs}} / P_{\text {in }}\right)$ at a wavelength of $450 \mathrm{~nm}$, measured while scanning over the area shown in the SEM image using the $3 \mathrm{D}$ piezoelectric stage. In the center of the wire, a peak in absorptance is visible, which corresponds to resonantly enhanced absorption via a Mie (geometrical) resonance. Mie 

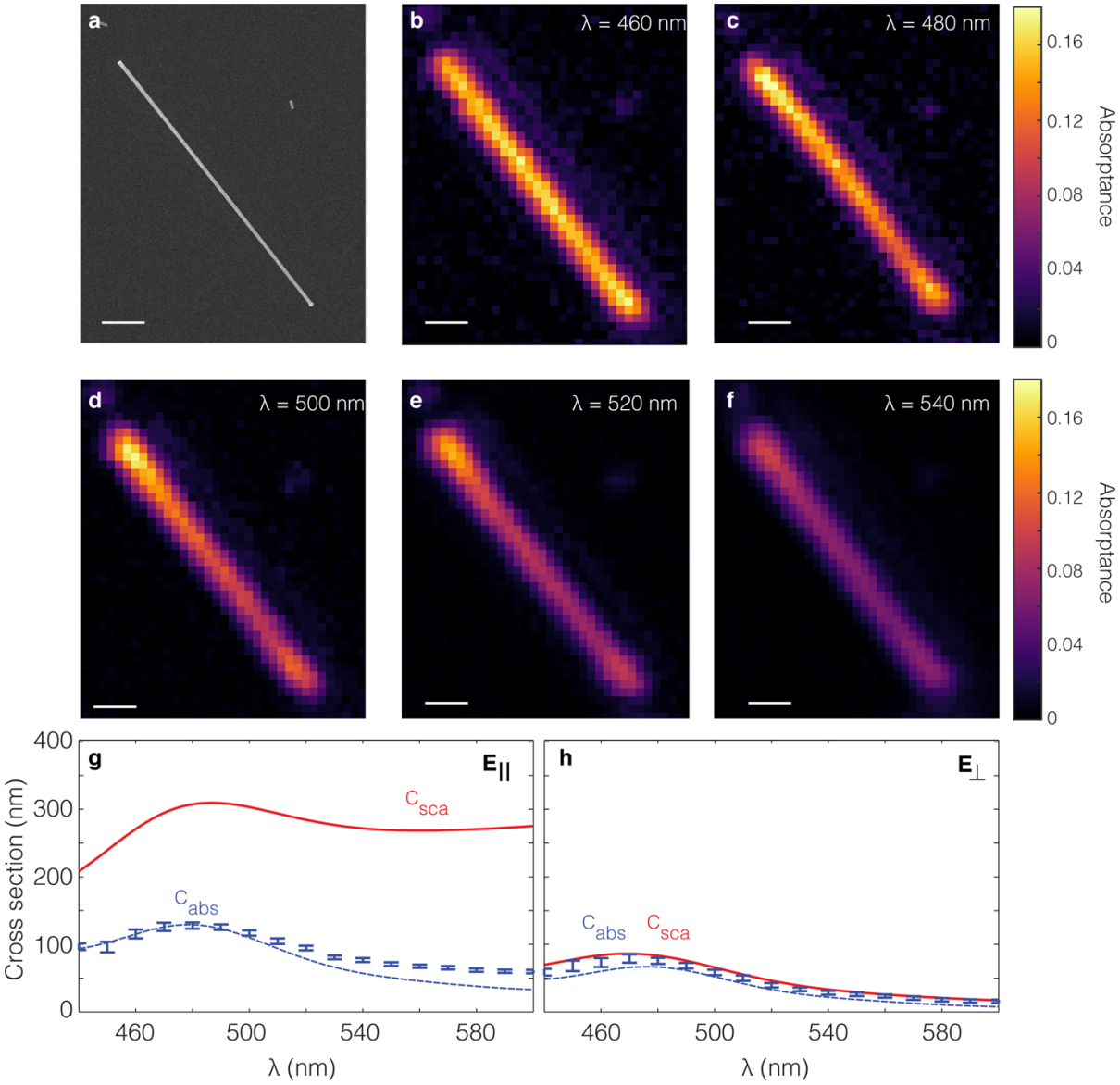

Figure 4. Absorption measurements of a GaAs nanowire. (a) SEM image of the GaAs nanowire. The scale bar is $1 \mu \mathrm{m}$. (b-f) Absorption maps from 460 to $540 \mathrm{~nm}$. Due to the smaller diameter of the nanowire and the lower tapering angle, the resonances appear to be less confined along the length of the nanowire. $(\mathrm{g}, \mathrm{h})$ Absorption spectra with the electric field parallel $(\mathrm{g})$ and perpendicular $(\mathrm{h})$ to the nanowire axis. The solid red and dashed blue lines show full-wave simulations of the scattering and absorption cross sections for a GaAs nanowire with the same diameter $(110 \mathrm{~nm})$.

resonances shift to longer wavelengths as the radius of the wire is increased, as they occur for approximately fixed values of the product $n k_{0} r$, where $n, k_{0}$, and $r$ are the refractive index, free space wavenumber, and radius, respectively. ${ }^{28,29}$ The absorption maps in Figure $2 \mathrm{c}-\mathrm{f}$ show the peak moving from smaller to larger diameter along the tapered nanowire as the illumination wavelength increases, providing a direct visualization of these diameter-dependent resonance phenomena.

Figure $2 \mathrm{~g}$ plots absorption intensity line cuts along the tapered nanowire in Figure $2 \mathrm{c}-\mathrm{f}$, together with calculated dispersion relations to identify the relevant Mie resonances. ${ }^{30}$ These resonances can be specified with the notation $\mathrm{TM}_{m l}$, where TM indicates the magnetic field is transverse to the long axis of the nanowire; $m$ is the azimuthal mode number, corresponding to the number of field maxima around the nanowire circumference; and $l$ indicates the number of radial field maxima. From these calculations shown in Figure $2 \mathrm{~g}$, it is clear that we observe the $\mathrm{TM}_{21}$ and $\mathrm{TM}_{12}$ resonances. These resonances are nearly degenerate and hard to disentangle spatially.

In addition to measuring nanoscale absorption maps at a fixed wavelength, we can also fix the laser position and measure local absorption spectra. Figure 3a shows an SEM image of the same nanowire (but rotated) and indicates two positions where spectra were measured. Figure $3 b-e$ shows the wavelengthdependent absorption cross sections of the nanowire at the two different positions, with the laser polarized either along or orthogonal to the nanowire axis. A geometric scaling factor is used to convert from absorptance to cross section: $C_{\mathrm{abs}}=A \sqrt{\pi w_{0}^{2} / 2}$, where $A$ is absorptance and $w_{0}$ is the beam radius. This factor can be derived by assuming that the nanowire is infinitely thin and then integrating the incident intensity along the nanowire. ${ }^{16}$ While the nanowire in reality is not infinitely thin, agreement with full-wave simulations is good (see Figure $3 \mathrm{~b}-\mathrm{e}$ ). However, because the scattered power significantly exceeds the absorption by up to a factor of 75 , the disagreement between the measurement and full-wave simulations is more likely due to the small fraction of scattered light that is not detected.

Due to the indirect band gap of silicon, these nanowires scatter very strongly, therefore providing a particularly challenging test case. Agreement with theory is better for more strongly absorbing materials such as GaAs, as we show in Figure 4. Figure 4a shows an SEM image of a GaAs nanowire with a radius ranging from $110 \mathrm{~nm}$ at the top of the image to 84 $\mathrm{nm}$ at the bottom. Figure $4 \mathrm{~b}-\mathrm{f}$ shows absorption maps for different wavelengths, just as for the silicon nanowire. However, since this nanowire has a lower tapering angle, the resonances do not appear localized along the nanowire length. Additionally, the nanowire has a smaller diameter, which means that a lower-order resonance is excited (the $\mathrm{TM}_{11}$ resonance), which 
has a broader linewidth and, interestingly, therefore also a larger spatial extent in a tapered nanowire than a resonance with a narrower linewidth would have. Figure $4 \mathrm{~g}$,h shows the cross section versus wavelength, compared to full-wave simulations of the nanowire absorption and scattering cross section for parallel and perpendicular incident polarizations, measured at the top of the wire. Both spectra show a resonance at $480 \mathrm{~nm}$, which are the $\mathrm{TM}_{11}$ and $\mathrm{TE}_{01}$ resonances in the parallel and perpendicular polarization, respectively. Since GaAs has a direct band gap, the scattering cross section is reduced and the absorption cross section is increased, indeed improving the agreement with numerical predictions.

Due to the linear extent of the nanowire, the intersection with the focused spot is significant and measurements above the noise floor are therefore easily achieved. To probe the detection limits of our setup, we also investigate small spherical gold nanospheres with a plasmon resonance near $500 \mathrm{~nm}$. While the absorptance of the nanowires reaches $>10 \%$ easily, the absorptance of a $60 \mathrm{~nm}$ diameter gold sphere will lie below $1 \%$ due to the small absorption cross section. Since the nanoparticle is localized in three dimensions, we now obtain for the absorptance (assuming that the nanoparticle can be treated as a point) $A=\sigma_{\text {abs }} I(x, y)$, where $I(x, y)$ is the intensity in the Gaussian spot. If the nanoparticle lies exactly in the focus, we obtain $\sigma_{\text {abs }}=A \times \pi w_{0}^{2} / 2$, where we used $I(x=0, y=0)=2 P_{\text {in }} /$ $\pi w_{0}^{2}$ and $A=P_{\text {abs }} / P_{\text {in }}$. This absorption cross section is shown in Figure $5 \mathrm{a}$ for a $60 \mathrm{~nm}$ diameter gold sphere and in Figure $5 \mathrm{~b}$ for a $200 \mathrm{~nm}$ diameter gold sphere. Even though the absorptance peaks at $0.5 \%$ for the $60 \mathrm{~nm}$ diameter nanosphere, the noise floor is low enough for accurate determination of the
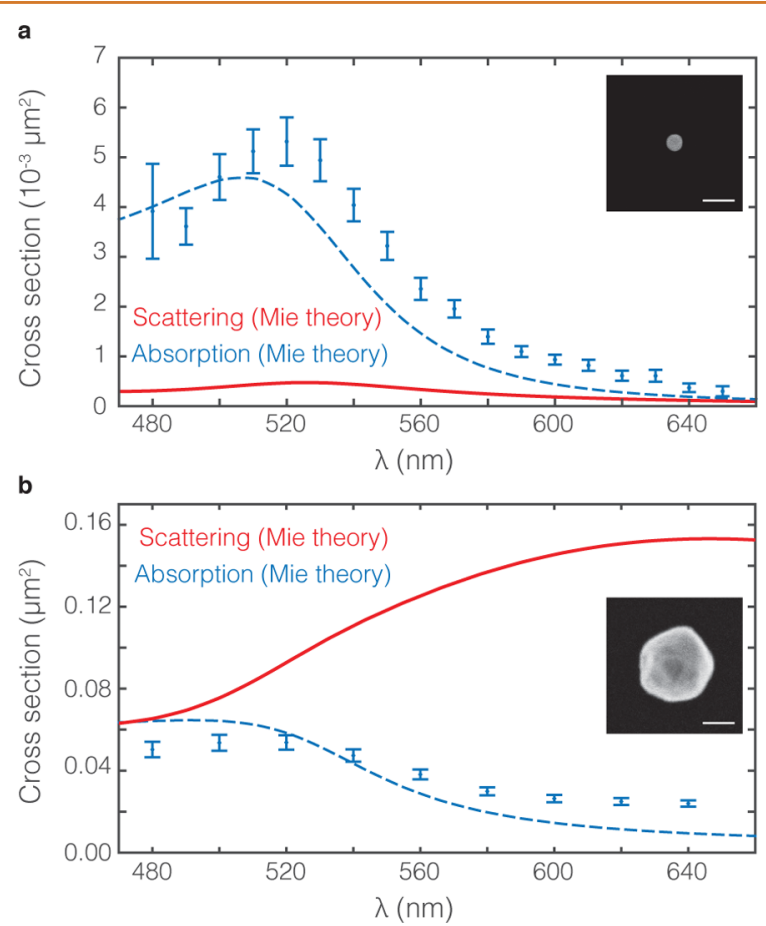

Figure 5. Absorption spectra of Au nanoparticles. (a) Absorption spectrum of a $60 \mathrm{~nm}$ Au nanoparticle compared to Mie theory predictions for absorption (dashed blue line) and scattering (red line). (b) Absorption spectrum of a $200 \mathrm{~nm} \mathrm{Au}$ nanoparticle compared to Mie theory calculations for absorption and scattering (dashed blue and red lines, respectively). The insets in both figures show representative gold particles along with a $100 \mathrm{~nm}$ scale bar. absorption cross section. In fact, the error margin shown in Figure 5 arises largely due to the determination of the Gaussian beam radius $w_{0}$.

Even for the gold sphere of $60 \mathrm{~nm}$, the signal-to-noise ratio is $\sim 30$, implying that smaller absorption cross sections can be measured. The noise floor depends on the laser characteristics, photodetectors, and detected optical power. While not the case in this experiment, the noise floor could ultimately be determined by photon shot noise. This would enable detection of absorption cross sections only a few square nanometers large, similar to extinction spectroscopy. ${ }^{12,31}$

\section{CONCLUSIONS}

To conclude, we have introduced a technique to perform quantitative and spatially resolved absorption spectroscopy on single scattering nanoparticles. The technique uses an integrating sphere to measure directly the transmitted, reflected, and scattered power, allowing for determination of the absorbed power. Calibration of the absorption measurement is very straightforward, and no assumptions on the nature of the scattering particle are required. We demonstrated integrating sphere microscopy by mapping the absorption cross section of tapered silicon and GaAs nanowires. These are essential characteristics for many optoelectronic devices, in particular, for light-emitting diodes and solar cells, but cannot be measured on the nanoscale with photothermal spectroscopy or any other known method. We therefore believe that this extension of the standard integrating sphere measurement to nanoscale systems can be of great utility in nanoscale optoelectronics, single nanoparticle spectroscopy, and other fields where quantitative and high spatial resolution measurements are desired.

\section{METHODS}

Experimental Setup. The light source in the experiment was a supercontinuum laser (Fianium WL-SC-390-3), which was made monochromatic using an acousto-optical tunable filter (AOTF, Crystal Technologies, with roughly $4 \mathrm{~nm}$ bandwidth). Power in the focused laser beam is on the order of several microwatts. The long working distance objective is a $17 \mathrm{~mm}$ working distance Mitutoyo M Apo Plan NIR 50× with NA $=0.42$. The integrating sphere is a LabSphere GPS020-SL, modified so that it can accommodate our objective lens. The photodetectors are Thorlabs amplified Si detectors (PDA100A), read out by Stanford Research Systems SR830 lock-in amplifiers. For the $\mathrm{Au}$ nanoparticle measurements the IS photodetector was replaced by a passive Newport 818-UV photodetector, which has a lower noise level than the Thorlabs transimpedance amplifiers. The transmission of the AOTF was digitally modulated at $20 \mathrm{kHz}$ with a $50 \%$ duty cycle for the nanowire measurements and at $700 \mathrm{~Hz}$ for the $\mathrm{Au}$ nanoparticle measurements. The sample holder was mounted on a Piezojena Tritor400 3D piezoelectric stage for high-resolution scanning of the sample, while for rough alignment, the piezoelectric stage was mounted on a Newport mechanical stage.

Sample Preparation. The silicon nanowires are grown epitaxially on a silicon substrate by the vapor-liquid-solid growth mechanism using an atmospheric pressure chemical vapor deposition system. The $40 \mathrm{~nm}$ gold colloids were used as catalysts. The growth was conducted at $800{ }^{\circ} \mathrm{C}$ for $5 \mathrm{~min}$ with silicon tetrachloride $\left(\mathrm{SiCl}_{4}\right)$ as the precursor. The carrier gas flows during growth were $\mathrm{Ar}=200 \mathrm{sccm}$ and $\mathrm{H}_{2}=50$ sccm, while only $75 \mathrm{sccm}$ Ar gas flows directly through the $\mathrm{SiCl}_{4}$ precursor bubbler (held at $0{ }^{\circ} \mathrm{C}$ in a temperature-controlled bath). Self-catalyzed GaAs nanowires were grown by solid-source III-V molecular beam epitaxy (MBE) directly on p-type $\mathrm{Si}(100)$ substrates by solid-source III-V Veeco MBE GEN-930. The NWs were grown with a Ga beam equivalent pressure, V/III flux ratio, substrate temperature, and growth duration of $8.7 \times 10^{-8}$ Torr, 50, $\sim 630^{\circ} \mathrm{C}$, 
and $1 \mathrm{~h}$, respectively. The substrate temperature was measured by a pyrometer. The gold nanoparticles (60 and $200 \mathrm{~nm}$ diameter) were acquired from BBI solutions.

Simulations. The nanowire cross section simulations were performed with Lumerical FDTD. The nanowire had a hexagonal shape and was assumed to be infinitely long (simulations were 2D). The refractive index of silicon and GaAs was based on tabulated data from Palik. ${ }^{32}$ In both the calculations of the dispersion diagrams and simulations, the parallel momentum was assumed to be zero, treating the wire as if illuminated under normal incidence. Considering the low effective NA in the experiment, this is an accurate assumption.

\section{ASSOCIATED CONTENT}

\section{S Supporting Information}

The Supporting Information is available free of charge on the ACS Publications website at DOI: 10.1021/acsnano.6b06534.

Technical details on the integrating sphere and sample holder, data analysis procedure details, and all reflection and integrating sphere signals corresponding to the figures in the main text (PDF)

\section{AUTHOR INFORMATION}

\section{Corresponding Author}

*E-mail: garnett@amolf.nl.

\section{ORCID}

Sander A. Mann: 0000-0002-7742-0615

\section{Notes}

The authors declare no competing financial interest.

\section{ACKNOWLEDGMENTS}

The authors are grateful to Ewold Verhagen and Ruben Maas for critical reading of the manuscript, Henk-Jan Boluijt, Mohamed Tachikirt, Marco Seynen, and the mechanical workshop at AMOLF for help with custom parts of the experimental setup. This work is part of the research programme of The Netherlands Organisation for Scientific Research (NWO). The research leading to these results has received funding from the European Research Council under the European Union's Seventh Framework Programme (FP/ 2007-2013)/ERC Grant Agreement No. 337328, "NanoEnabledPV". H.L. and Y.Z. acknowledge the support of Leverhulme Trust. H.L. would like to thank The Royal Society for funding his University Research Fellowship.

\section{REFERENCES}

(1) Crut, A.; Maioli, P.; Del Fatti, N.; Vallée, F. Optical Absorption and Scattering Spectroscopies of Single Nano-Objects. Chem. Soc. Rev. 2014, 43, 3921.

(2) Zijlstra, P.; Orrit, M. Single Metal Nanoparticles: Optical Detection, Spectroscopy and Applications. Rep. Prog. Phys. 2011, 74, 106401.

(3) Olson, J.; Dominguez-medina, S.; Hoggard, A.; Wang, L.; Chang, W.; Link, S. Optical Characterization of Single Plasmonic Nanoparticles. Chem. Soc. Rev. 2015, 44, 40-57.

(4) Yorulmaz, M.; Nizzero, S.; Hoggard, A.; Wang, L.-Y.; Cai, Y.-Y.; Su, M.-N.; Chang, W.-S.; Link, S. Single-Particle Absorption Spectroscopy by Photothermal Contrast. Nano Lett. 2015, 15, 3041-3047.

(5) Gaiduk, A.; Yorulmaz, M.; Ruijgrok, P. V.; Orrit, M. RoomTemperature Detection of a Single Molecule's Absorption by Photothermal Contrast. Science 2010, 330, 353-356.

(6) Baffou, G.; Bon, P.; Savatier, J.; Polleux, J.; Zhu, M.; Merlin, M.; Rigneault, H.; Monneret, S. Thermal Imaging of Nanostructures by Quantitative Optical Phase Analysis. ACS Nano 2012, 6, 2452-2458.
(7) Berto, P.; Ureña, E. B.; Bon, P.; Quidant, R.; Rigneault, H.; Baffou, G. Quantitative Absorption Spectroscopy of Nano-Objects. Phys. Rev. B: Condens. Matter Mater. Phys. 2012, 86, 165417.

(8) Arbouet, A.; Christofilos, D.; Del Fatti, N.; Vallée, F.; Huntzinger, J. R.; Arnaud, L.; Billaud, P.; Broyer, M. Direct Measurement of the Single-Metal-Cluster Optical Absorption. Phys. Rev. Lett. 2004, 93, 127401.

(9) Muskens, O. L.; Bachelier, G.; Del Fatti, N.; Vallée, F.; Brioude, A.; Jiang, X.; Pileni, M. P. Quantitative Absorption Spectroscopy of a Single Gold Nanorod. J. Phys. Chem. C 2008, 112, 8917-8921.

(10) Blancon, J.-C.; Paillet, M.; Tran, H. N.; Than, X. T.; Guebrou, S. A.; Ayari, A.; San Miguel, A.; Phan, N.-M.; Zahab, A.-A.; Sauvajol, J.-L.; Del Fatti, N.; Vallée, F. Direct Measurement of the Absolute Absorption Spectrum of Individual Semiconducting Single-Wall Carbon Nanotubes. Nat. Commun. 2013, 4, 2542.

(11) Husnik, M.; Linden, S.; Diehl, R.; Niegemann, J.; Busch, K.; Wegener, M. Quantitative Experimental Determination of Scattering and Absorption Cross-Section Spectra of Individual Optical Metallic Nanoantennas. Phys. Rev. Lett. 2012, 109, 233902.

(12) Celebrano, M.; Kukura, P.; Renn, A.; Sandoghdar, V. SingleMolecule Imaging by Optical Absorption. Nat. Photonics 2011, 5, 9598

(13) Brongersma, M. L.; Halas, N. J.; Nordlander, P. PlasmonInduced Hot Carrier Science and Technology. Nat. Nanotechnol. 2015, $10,25-34$.

(14) Clavero, C. Plasmon-Induced Hot-Electron Generation at Nanoparticle/metal-Oxide Interfaces for Photovoltaic and Photocatalytic Devices. Nat. Photonics 2014, 8, 95-103.

(15) Sciacca, B.; Mann, S. A.; Tichelaar, F. D.; Zandbergen, H. W.; van Huis, M. A.; Garnett, E. C. Solution-Phase Epitaxial Growth of Quasi-Monocrystalline Cuprous Oxide on Metal Nanowires. Nano Lett. 2014, 14, 5891-5898.

(16) Mann, S. A.; Oener, S. Z.; Cavalli, A.; Haverkort, J. E. M.; Bakkers, E. P. A. M.; Garnett, E. C. Quantifying Losses and Thermodynamic Limits in Nanophotonic Solar Cells. Nat. Nanotechnol. 2016, 11, 1071-1075.

(17) Burkhard, G. F.; Hoke, E. T.; McGehee, M. D. Accounting for Interference, Scattering, and Electrode Absorption to Make Accurate Internal Quantum Efficiency Measurements in Organic and Other Thin Solar Cells. Adv. Mater. 2010, 22, 3293-3297.

(18) Leyre, S.; Coutino-Gonzalez, E.; Joos, J. J.; Ryckaert, J.; Meuret, Y.; Poelman, D.; Smet, P. F.; Durinck, G.; Hofkens, J.; Deconinck, G.; Hanselaer, P. Absolute Determination of Photoluminescence Quantum Efficiency Using an Integrating Sphere Setup. Rev. Sci. Instrum. 2014, 85, 123115.

(19) Gargas, D. J.; Gao, H.; Wang, H.; Yang, P. High Quantum Efficiency of Band-Edge Emission from $\mathrm{ZnO}$ Nanowires. Nano Lett. 2011, 11, 3792-3796.

(20) Duan, X.; Huang, Y.; Cui, Y.; Wang, J.; Lieber, C. M. Indium Phosphide Nanowires as Building Blocks for Nanoscale Electronic and Optoelectronic Devices. Nature 2001, 409, 66-69.

(21) Krogstrup, P.; Jørgensen, H. I.; Heiss, M.; Demichel, O.; Holm, J. V.; Aagesen, M.; Nygard, J.; Fontcuberta i Morral, A. SingleNanowire Solar Cells beyond the Shockley-Queisser Limit. Nat. Photonics 2013, 7, 306-310.

(22) Wallentin, J.; Anttu, N.; Asoli, D.; Huffman, M.; Aberg, I.; Magnusson, M. H.; Siefer, G.; Fuss-Kailuweit, P.; Dimroth, F.; Witzigmann, B.; Xu, H. Q.; Samuelson, L.; Deppert, K.; Borgstrom, M. T. InP Nanowire Array Solar Cells Achieving 13.8\% Efficiency by Exceeding the Ray Optics Limit. Science 2013, 339, 1057-1060.

(23) Svensson, C. P. T.; Mårtensson, T.; Trägårdh, J.; Larsson, C.; Rask, M.; Hessman, D.; Samuelson, L.; Ohlsson, J. Monolithic GaAs/ InGaP Nanowire Light Emitting Diodes on Silicon. Nanotechnology 2008, 19, 305201.

(24) Minot, E. D.; Kelkensberg, F.; van Kouwen, M.; van Dam, J. A.; Kouwenhoven, L. P.; Zwiller, V.; Borgström, M. T.; Wunnicke, O.; Verheijen, M. A.; Bakkers, E. P. A. M. Single Quantum Dot Nanowire LEDs. Nano Lett. 2007, 7, 367-371. 
(25) van Dam, D.; Abujetas, D. R.; Paniagua-Domínguez, R.; Sánchez-Gil, J. A.; Bakkers, E. P. A. M.; Haverkort, J. E. M.; Gómez Rivas, J. Directional and Polarized Emission from Nanowire Arrays. Nano Lett. 2015, 15, 4557-4563.

(26) Huang, M. H.; Mao, S.; Feick, H.; Yan, H.; Wu, Y.; Kind, H.; Weber, E.; Russo, R.; Yang, P. Room-Temperature Ultraviolet Nanowire Nanolasers. Science 2001, 292, 1897-1899.

(27) Heiss, M.; Fontana, Y.; Gustafsson, A.; Wüst, G.; Magen, C.; O’Regan, D. D.; Luo, J. W.; Ketterer, B.; Conesa-Boj, S.; Kuhlmann, A. V.; Houel, J.; Russo-Averchi, E.; Morante, J. R.; Cantoni, M.; Marzari, N.; Arbiol, J.; Zunger, A.; Warburton, R. J.; Fontcuberta i Morral, A. Self-Assembled Quantum Dots in a Nanowire System for Quantum Photonics. Nat. Mater. 2013, 12, 439-444.

(28) Mann, S. A.; Garnett, E. C. Extreme Light Absorption in Thin Semiconductor Films Wrapped around Metal Nanowires. Nano Lett. 2013, 13, 3173-3178.

(29) Bohren, C. E.; Huffman, D. R. Absorption and Scattering of Light by Small Particles; John Wiley \& Sons, Inc.: New York, 1983.

(30) Yu, Y.; Cao, L. Coupled Leaky Mode Theory for Light Absorption in 2D, 1D, and 0D Semiconductor Nanostructures. Opt. Express 2012, 20, 13847.

(31) McDonald, M. P.; Vietmeyer, F.; Aleksiuk, D.; Kuno, M. Supercontinuum Spatial Modulation Spectroscopy: Detection and Noise Limitations. Rev. Sci. Instrum. 2013, 84, 113104.

(32) Palik, E. D. Handbook of Optical Constants of Solids; Elsevier Academic Press: Waltham, MA, 1985. 